\title{
ESTIMATES OF THE BERGMAN DISTANCE ON DINI-SMOOTH BOUNDED PLANAR DOMAINS
}

\author{
NIKOLAI NIKOLOV AND MARIA TRYBUŁA
}

\begin{abstract}
Precise estimates for the Bergman distances of Dinismooth bounded planar domains are given. These estimates imply that on such domains the Bergman distance almost coincides with the Carathéodory and Kobayashi distances.
\end{abstract}

\section{REsults}

In [6, Proposition 8], the first named author found optimal estimates for Carathéodory and Kobayashi distances, $c_{D}$ and $k_{D}$, on Dini-smooth bounded planar domains $D$ in terms of $d_{D}=\operatorname{dist}(\cdot, \partial D)$. In this paper we shall prove similar estimates for the Bergman distance $b_{D}$. For convenience of the reader, the definitions of these three distances, as well as of Dini-smoothness, are given in the next section.

Proposition 1. Let D be a Dini-smooth bounded planar domain. Then there exists a constant $c>1$ such that

$$
\begin{aligned}
& \sqrt{2} \log \left(1+\frac{|z-w|}{c \sqrt{d_{D}(z) d_{D}(w)}}\right) \leq b_{D}(z, w) \\
\leq & \sqrt{2} \log \left(1+\frac{c|z-w|}{\sqrt{d_{D}(z) d_{D}(w)}}\right), \quad z, w \in D .
\end{aligned}
$$

By [6, Proposition 8], the same result holds for $\sqrt{2} c_{D}$ and $\sqrt{2} k_{D}$ instead of $b_{D}$. So, we have the following

2010 Mathematics Subject Classification. 32A25, 32F45.

Key words and phrases. Bergman, Carathéodory and Kobayashi distances; Dinismooth planar domain.

M. Trybuła is partially supported by the International $\mathrm{PhD}$ programme "Geometry and Topology in Physical Models" of the Foundation for Polish Science, by the Polish National Science Center - grant PRO-2013/11/N/ST1/03609, and by the Bulgarian National Science Found under contract DFNI-I 02/14. The initial version of this paper was prepared during her visit to the Institute of Mathematics and Informatics, Bulgarian Academy of Science, October 2013 - April 2014 and July 2014. 
Corollary 2. If $D$ is a Dini-smooth bounded planar domain, then the differences $b_{D}-\sqrt{2} c_{D}$ and $b_{D}-\sqrt{2} k_{D}$ are bounded.

Note that Proposition 1 is equivalent to

Proposition $\mathbf{1}^{\prime}$. Let $D$ be a Dini-smooth bounded planar domain. There exists a constant $c>1$ such that:

- if $|z-w|^{2}>d_{D}(z) d_{D}(w)$, then

$$
\log \frac{|z-w|^{2}}{d_{D}(z) d_{D}(w)}-c<\sqrt{2} b_{D}(z, w)<\log \frac{|z-w|^{2}}{d_{D}(z) d_{D}(w)}+c ;
$$

- if $|z-w|^{2} \leq d_{D}(z) d_{D}(w)$, then

$$
\frac{|z-w|}{c \sqrt{d_{D}(z) d_{D}(w)}} \leq b_{D}(z, w) \leq \frac{c|z-w|}{\sqrt{d_{D}(z) d_{D}(w)}} .
$$

Remark. (a) The Dini-smoothness is essential as an example of a $\mathcal{C}^{1}$-smooth bounded simply connected planar domain shows (see [8, Example 2]).

(b) One of the missing properties of $b_{D}$ in comparison with $c_{D}$ and $l_{D}$ is monotonicity under inclusion of (planar) domains. However, the invariants $M_{D}$ and $K_{D}$ share this property which allows us to modify the approach from [6].

(c) Results in $\mathbb{C}^{n}$ in the spirit of Proposition 1 and Corollary 3 can be found in [1] and [7], respectively, where the strictly pseudoconvex domains are treated. Note also that the Levi pseudoconvex corank one domains are considered in [3]. As can be expected, our estimates are more precise than those in [1] and [3, when the two points, $z$ and $w$, are close to each other.

(d) It follows by the second statement of Proposition $1^{\prime}$ that

$$
\frac{1}{c d_{D}(u)} \leq \liminf _{\substack{z, w \rightarrow u \\ z \neq w}} \frac{b_{D}(z, w)}{|z-w|}, \quad u \in D .
$$

This inequality agrees with the fact that (cf. [5, Lemma 4.3 .3 (e)])

$$
\limsup _{\substack{z, w \rightarrow u \\ z \neq w}} \frac{b_{D}(z, w)}{|z-w|} \leq \beta_{D}(u ; 1)
$$

(cf. [5, Lemma 4.3.3 (e)]) and the equality (see [4, Remark, p. 11])

$$
\lim _{u \rightarrow \partial D} \beta_{D}(u ; 1) d_{D}(u)=\frac{\sqrt{2}}{2} .
$$


Recall now another comparison result between $c_{D}$ and $k_{D}$ (see [6], Proposition 9]): if $D$ is a finitely connected bounded planar domain without isolated boundary points 11 then

$$
\lim _{\substack{w \rightarrow \partial D \\ z \neq w}} \frac{c_{D}(z, w)}{k_{D}(z, w)}=1 \quad \text { uniformly in } z \in D .
$$

Similar results for $c_{D}, k_{D}, l_{D}$ and $b_{D}$ in the strictly pseudoconvex case can be found in [9, Theorem 1] and [7, Proposition 4].

The next proposition shows that (11) remains true if we replace $c_{D}$ or $k_{D}$ by $b_{D} / \sqrt{2}$.

Proposition 3. If $D$ is a finitely connected bounded planar domain without isolated boundary points, then

$$
\lim _{\substack{w \rightarrow \partial D \\ z \neq w}} \frac{b_{D}(z, w)}{c_{D}(z, w)}=\lim _{\substack{w \rightarrow \partial D \\ z \neq w}} \frac{b_{D}(z, w)}{k_{D}(z, w)}=\sqrt{2} \quad \text { uniformly in } z \in D .
$$

Remark. The isolated boundary points condition is essential. Indeed, if $p$ is an isolated boundary point of a planar domain $D \neq \mathbb{C} \backslash\{p\}$, then $c_{D}=c_{D \cup\{p\}}$ and $b_{D}=b_{D \cup\{p\}}$, but $k_{D}(z, w) \rightarrow \infty$ as $w \rightarrow p$ and $z \in D$ is fixed.

\section{Definitions}

1. A boundary point $p$ of a planar domain $D$ is said to be Dini-smooth if $\partial D$ near $p$ is given by a Dini-smooth curve $\gamma:[0,1] \rightarrow \mathbb{C}$ with $\gamma^{\prime} \neq 0$ (i.e., $\int_{0}^{1} \frac{\omega(t)}{t} d t<\infty$, where $\omega$ is the modulus of continuity of $\gamma^{\prime}$ ). A planar domain is called Dini-smooth if all its boundary points are Dini-smooth.

2. Let $D$ be a domain in $\mathbb{C}^{n}$.

The Bergman distance $b_{D}$ of $D$ is the integrated form of the Bergman metric $\beta_{D}$, i.e.,

$$
b_{D}(z, w)=\inf _{\gamma} \int_{0}^{1} \beta_{D}\left(\gamma(t) ; \gamma^{\prime}(t)\right) d t, \quad z, w \in D,
$$

where the infimum is taken over all smooth curves $\gamma:[0,1] \rightarrow D$ with $\gamma(0)=z$ and $\gamma(1)=w$.

Recall that

$$
\beta_{D}(z ; X)=\frac{M_{D}(z ; X)}{K_{D}(z)}, \quad z \in D, X \in \mathbb{C}^{n},
$$

\footnotetext{
${ }^{1}$ Any $C^{1}$-smooth bounded planar domain is such a domain.
} 
where

$$
M_{D}(z ; X)=\sup \left\{\left|f^{\prime}(z) X\right|: f \in L_{h}^{2}(D),\|f\|_{L^{2}(D)} \leq 1, f(z)=0\right\}
$$

and

$$
K_{D}(z)=\sup \left\{|f(z)|: f \in L_{h}^{2}(D),\|f\|_{L^{2}(D)} \leq 1\right\}
$$

is the square root of the Bergman kernel on the diagonal (we assume that $K_{D}>0$; for example, this holds if $D$ is bounded).

The Carathéodory distance $c_{D}$ and the Lempert function $l_{D}$ of $D$ are defined as follows:

$$
\begin{gathered}
c_{D}(z, w)=\sup \left\{\tanh ^{-1}|f(w)|: f \in \mathcal{O}(D, \mathbb{D}), \text { with } f(z)=0\right\}, \\
l_{D}(z, w)=\inf \left\{\tanh ^{-1}|\alpha|: \exists \varphi \in \mathcal{O}(\mathbb{D}, D) \text { with } \varphi(0)=z, \varphi(\alpha)=w\right\},
\end{gathered}
$$

where $\mathbb{D}$ is the unit disc.

The Kobayashi distance $k_{D}$ is the largest pseudodistance not exceeding $l_{D}$. It is well-known that $k_{D}$ is the integrated form of Kobayashi metric $\kappa_{D}$ defined by

$$
\kappa_{D}(z ; X)=\inf \left\{|\alpha|: \exists \varphi \in \mathcal{O}(\mathbb{D}, D) \text { with } \varphi(0)=z, \alpha \varphi^{\prime}(0)=X\right\} .
$$

Note that $c_{D} \leq k_{D} \leq l_{D}$ and $c_{D} \leq b_{D}$. On the other hand, $k_{D}=l_{D}$ for any planar domain $D$ (cf. [5, Remark 3.3.8(e)]).

We refer to [5] for other basic properties of the above invariants.

\section{Proofs}

To prove Proposition 1, we shall need the following

Lemma 4. (a)

$$
\begin{aligned}
& \log \left(1+\frac{|z-w|}{\sqrt{\left(1-|z|^{2}\right)\left(1-|w|^{2}\right)}}\right) \leq \frac{b_{\mathbb{D}}(z, w)}{\sqrt{2}} \\
& \leq \log \left(1+\frac{2|z-w|}{\sqrt{\left(1-|z|^{2}\right)\left(1-|w|^{2}\right)}}\right) ;
\end{aligned}
$$

(b)

$$
\log \left(1+\frac{|z-w|}{2 \sqrt{d_{\mathbb{D}}(z) d_{\mathbb{D}}(w)}}\right) \leq \frac{b_{\mathbb{D}}(z, w)}{\sqrt{2}} \leq \log \left(1+\frac{\sqrt{2}|z-w|}{\sqrt{d_{\mathbb{D}}(z) d_{\mathbb{D}}(w) .}}\right) .
$$

Proof. (a) We have that

$$
\sqrt{2} b_{\mathbb{D}}(z, w)=2 k_{\mathbb{D}}(z, w)=\log \frac{1+\left|\frac{z-w}{1-\bar{z} w}\right|}{1-\left|\frac{z-w}{1-\bar{z} w}\right|}=
$$


ESTIMATES OF THE BERGMAN DISTANCE ON PLANAR DOMAINS 5

$\log \left(1+\frac{2|z-w|}{|1-\bar{z} w|-|z-w|}\right)=\log \left(1+2|z-w| \frac{|1-\bar{z} w|+|z-w|}{\left(1-|z|^{2}\right)\left(1-|w|^{2}\right)}\right)$.

It remains to use that

$$
|1-\bar{z} w|^{2}=\left(1-|z|^{2}\right)\left(1-|w|^{2}\right)+|z-w|^{2}
$$

and hence

$$
\sqrt{\left(1-|z|^{2}\right)\left(1-|w|^{2}\right)} \leq|1-\bar{z} w| \leq \sqrt{\left(1-|z|^{2}\right)\left(1-|w|^{2}\right)}+|z-w|
$$

(b) The lower estimate follows from (a) and $d_{\mathbb{D}}(z)=1-|z| \geq \frac{1-|z|^{2}}{2}$.

To get the upper estimate, we have to show that

$$
1+2|z-w| \frac{|1-\bar{z} w|+|z-w|}{\left(1-|z|^{2}\right)\left(1-|w|^{2}\right)} \leq\left(1+\frac{\sqrt{2}|z-w|}{\sqrt{(1-|z|)(1-|w|)}}\right)^{2}
$$

which is equivalent to

$$
\frac{|1-\bar{z} w|+|z-w|}{\left(1-|z|^{2}\right)\left(1-|w|^{2}\right)} \leq \frac{\sqrt{2}}{\sqrt{(1-|z|)(1-|w|)}}+\frac{|z-w|}{(1-|z|)(1-|w|)}
$$

i.e.,

$|1-\bar{z} w| \leq \sqrt{2(1-|z|)(1-|w|)}(1+|z|)(1+|w|)+|z-w|(|z|+|w|+|z w|)$.

So, it is enough to prove that

$|1-\bar{z} w|^{2} \leq 2(1-|z|)(1-|w|)(1+|z|)^{2}(1+|w|)^{2}+|z-w|^{2}(|z|+|w|+|z w|)^{2}$.

Using (21) and dividing by $(1+|z|)(1+|w|)$, the last inequality becomes $|z-w|^{2}(1-|z|-|w|-|z w|) \leq(1-|z|-|w|+|z w|)(1+2|z|+2|w|+2|z w|)$ which follows from $|z-w|^{2} \leq|z|^{2}+|w|^{2}+2|z w| \leq|z|+|w|+2|z w|$.

Remark. (a) The constants 1 and 2 in front of $|z-w|$ in the lower and upper estimates in Lemma 4 (a) are sharp. To see this, let

$$
\frac{|z-w|^{2}}{\left(1-|z|^{2}\right)\left(1-|w|^{2}\right)} \rightarrow 0 \text { and } \infty \text {, respectively. }
$$

(b) The constants $\frac{1}{2}$ and $\sqrt{2}$ in front of $|z-w|$ in the lower and upper estimates in Lemma 4 (b) are sharp, too. To see this, let $|z| \rightarrow 1$ and then

$$
\frac{|z-w|^{2}}{\left(1-|z|^{2}\right)\left(1-|w|^{2}\right)} \rightarrow 0 \text { and } w \rightarrow 0, \text { respectively. }
$$

Proof of Proposition 1. Let $D \supset\left(z_{n}\right) \rightarrow p$ and $D \supset\left(w_{n}\right) \rightarrow q\left(z_{n} \neq\right.$ $\left.w_{n}\right)$. It is enough to find a constant $c>1$ such that the respective estimates for $b_{D}\left(z_{n}, w_{n}\right)$ hold for any $n$. 
Note that, by [6, Proposition 5 and Corollary 6], for any neighborhood $U$ of $p$ there exist a neighborhood $V \subset U$ and a constant $c_{1}>0$ such that

(3) $\left|\sqrt{2} b_{D}(z, w)+\log d_{D}(z)+\log d_{D}(w)\right|<c_{1}, \quad z \in D \cap V, w \in D \backslash U$.

This inequality provides the desired constant if $D \ni p \neq q \in D$, or $p \in \partial D, q \in D$, or $p \in D, q \in \partial D$, or $\partial D \ni p \neq q \in \partial D$.

For a planar domain $\Omega$, set $\beta_{\Omega}(z)=\beta_{\Omega}(z ; 1), M_{\Omega}(z)=M_{\Omega}(z ; 1)$ and $\kappa_{\Omega}(z)=\kappa_{\Omega}(z ; 1)$.

If $p=q \in D$, then the continuity of $\beta_{D}$ implies that

$$
\frac{b_{D}\left(z_{n}, w_{n}\right)}{\left|z_{n}-w_{n}\right|} \rightarrow \beta_{D}(p)>0
$$

and we may easily find the desired constant.

It remains to consider the most difficult case $p=q \in \partial D$. Some of our arguments will be close to that in the proof of [6, Proposition 5].

This proof allows us to assume that $p=1$ and

$$
\{z \in \mathbb{D}:|z-1|<r\}=: E_{r} \subset D \subset \mathbb{D}
$$

for some $r>0$ (after an appropriate conformal map). Then

$$
\sqrt{2} \frac{\kappa_{\mathbb{D}}^{2}(z)}{\kappa_{E_{r}}(z)}=\frac{M_{\mathbb{D}}(z)}{K_{E_{r}}(z)} \leq \beta_{D}(z) \leq \frac{M_{E_{r}}(z)}{K_{\mathbb{D}}(z)}=\sqrt{2} \frac{\kappa_{E_{r}}^{2}(z)}{\kappa_{\mathbb{D}}(z)}, \quad z \in E_{r}
$$

(the both equalities hold because $E_{r}$ is a simply connected domain).

Fix an $r_{1} \in(0, r)$. The localization of the Kobayashi metrics from [2, Theorem 2.1 and Lemma 2.2] implies that

$$
\kappa_{\mathbb{D}}(z)>\left(1-c_{2} d_{\mathbb{D}}(z)\right) \kappa_{E_{r}}(z), \quad z \in E_{r_{1}},
$$

for some constant $c_{2}>0$. Choose an $r_{2} \in\left(0, r_{1}\right]$ with $2 c_{2} r_{2} \leq 1$. Then

$$
\left(1-c_{2} d_{\mathbb{D}}(z)\right) \kappa_{\mathbb{D}}(z)<\frac{\beta_{D}(z)}{\sqrt{2}}<\left(1+2 c_{2} d_{\mathbb{D}}(z)\right) \kappa_{\mathbb{D}}(z), \quad z \in E_{r_{2}} .
$$

Since $\kappa_{\mathbb{D}}(z)=\frac{\beta_{\mathbb{D}}(z)}{\sqrt{2}}=\frac{1}{1-|z|^{2}}$, it follows for $c_{3}=2 \sqrt{2} c_{2}$ that

$$
\frac{1}{\sqrt{2}}\left(\frac{1}{d_{\mathbb{D}}(z)}-c_{2}\right)<\beta_{D}(z)<\beta_{\mathbb{D}}(z)+c_{3}, \quad z \in E_{r_{2}} .
$$

We may assume that $z_{n}, w_{n} \in E_{r_{3}}$, where $r_{3} \in\left(0, r_{2} / 2\right]$ is such that if $\alpha_{n}$ is the shorter arc with endpoints $z_{n}$ and $w_{n}$ of the circle through $z_{n}$ and $w_{n}$ which is orthogonal to the unit circle, then $\alpha_{n} \subset E_{r_{2}}$. Hence

$$
b_{D}\left(z_{n}, w_{n}\right)<\int_{\alpha_{n}}\left(\frac{\sqrt{2}}{1-|z|^{2}}+c_{3}\right) d l
$$




$$
=b_{\mathbb{D}}\left(z_{n}, w_{n}\right)+c_{3} l\left(\alpha_{n}\right)<b_{\mathbb{D}}\left(z_{n}, w_{n}\right)+2 c_{3}\left|z_{n}-w_{n}\right|
$$

for any $n$.

Now, using Lemma 4 (b) and the equality

$$
d_{\mathbb{D}}(z)=d_{D}(z), \quad z \in E_{r_{3}}
$$

it is easy to find a constant $c>1$ such that the upper estimate for $b_{D}\left(z_{n}, w_{n}\right)$ in Proposition 1 holds for any $n$.

It is left to manage the lower estimate. Let $\gamma_{n}:[0,1] \rightarrow D$ be a smooth curve such that $\gamma_{n}(0)=z_{n}, \gamma_{n}(1)=w_{n}$ and

$$
b_{D}\left(z_{n}, w_{n}\right)+\left|z_{n}-w_{n}\right|>\int_{0}^{1} \beta_{D}\left(\gamma_{n}(t) ; \gamma_{n}^{\prime}(t)\right) d t .
$$

Consider the set $A$ of all $n$ for which $\gamma_{n}(0,1) \not \subset E_{r_{2}}$. For any $n \in A$ we may find a number $t_{n} \in(0,1)$ such that $\left|u_{n}-1\right|=r_{2}$, where $u_{n}=\gamma\left(t_{n}\right)$. By (3), there exists a constant $c_{4}>0$, which does not depend on $n \in A$, such that

$$
\begin{gathered}
b_{D}\left(z_{n}, w_{n}\right)+\left|z_{n}-w_{n}\right|>b_{D}\left(z_{n}, u_{n}\right)+b_{D}\left(u_{n}, w_{n}\right) \\
>-\frac{\log d_{D}\left(z_{n}\right)}{\sqrt{2}}-\frac{\log d_{D}\left(w_{n}\right)}{\sqrt{2}}-c_{4} .
\end{gathered}
$$

This inequality easily provides a constant $c>1$ for which the lower estimate for $b_{D}\left(z_{n}, w_{n}\right)$ in Proposition 1 holds for any $n \in A$.

Let now $n \notin A$. Since

$$
\begin{gathered}
d_{\mathbb{D}}\left(\gamma_{n}(t)\right) \leq f_{n}(t):=d_{\mathbb{D}}\left(z_{n}\right)+\left|z_{n}-\gamma_{n}(t)\right|<2 r_{3}+r_{2} \leq 2 r_{2} \leq 1 / c_{2}, \\
d_{\mathbb{D}}\left(\gamma_{n}(t)\right) \leq g_{n}(t):=d_{\mathbb{D}}\left(w_{n}\right)+\left|w_{n}-\gamma_{n}(t)\right|<1 / c_{2}
\end{gathered}
$$

and $|s|^{\prime} \geq\left|s^{\prime}\right|$, it follows by (6) that, for any $t_{n} \in(0,1)$,

$$
\begin{gathered}
\sqrt{2}\left(b_{D}\left(z_{n}, w_{n}\right)+\left|z_{n}-w_{n}\right|\right)>\int_{0}^{1}\left(\frac{1}{d_{\mathbb{D}}\left(\gamma_{n}(t)\right)}-c_{2}\right)\left|\gamma_{n}^{\prime}(t)\right| d t \\
\geq \int_{0}^{t_{n}}\left(\frac{1}{f_{n}(t)}-c_{2}\right) d f_{n}(t)-\int_{t_{n}}^{1}\left(\frac{1}{g_{n}(t)}-c_{2}\right) d g_{n}(t) \\
=\log \left(1+\frac{\left|z_{n}-\gamma_{n}\left(t_{n}\right)\right|}{d_{\mathbb{D}}\left(z_{n}\right)}\right)-c_{2}\left|z_{n}-\gamma_{n}\left(t_{n}\right)\right| \\
+\log \left(1+\frac{\left|w_{n}-\gamma_{n}\left(t_{n}\right)\right|}{d_{\mathbb{D}}\left(w_{n}\right)}\right)-c_{2}\left|w_{n}-\gamma_{n}\left(t_{n}\right)\right| \\
>\log \left(1+\frac{\left|z_{n}-\gamma_{n}\left(t_{n}\right)\right| \cdot\left|w_{n}-\gamma_{n}\left(t_{n}\right)\right|}{c_{5} d_{\mathbb{D}}\left(z_{n}\right) d_{\mathbb{D}}\left(w_{n}\right)}\right)
\end{gathered}
$$

for some constant $c_{5}>1$. Choosing now $t_{n}$ such that $\left|z_{n}-\gamma_{n}\left(t_{n}\right)\right|=$ $\left|w_{n}-\gamma_{n}\left(t_{n}\right)\right|$ and using (7), we obtain the lower estimate in Proposition 1. 
So, Proposition 1 is completely proved.

Proof of Proposition 3. By the Köbe uniformization theorem, we may assume that $\partial D$ consists of disjoint circles. Using Proposition 1, Corollary 2, (11) and compactness, it is enough to prove that

$$
\lim _{\substack{z, w \rightarrow p \\ z \neq w}} \frac{b_{D}(z, w)}{k_{D}(z, w)}=\sqrt{2}
$$

for any point $p \in \partial D$.

Applying an inversion, we may assume that the outer boundary of $D$ is the unit circle and $p=1$. Then (4) and (5) imply

$$
\lim _{z \rightarrow 1} \frac{\beta_{E_{r}}(z)}{\beta_{D}(z)}=1=\lim _{z \rightarrow 1} \frac{\kappa_{E_{r}}(z)}{\kappa_{D}(z)} .
$$

The first equality shows that $\liminf _{\substack{z, w \rightarrow 1 \\ z \neq w}} \frac{b_{E_{r}}(z, w)}{b_{D}(z, w)} \geq 1$.

To get that

$$
\limsup _{\substack{z, w \rightarrow 1 \\ z \neq w}} \frac{b_{E_{r}}(z, w)}{b_{D}(z, w)} \leq 1
$$

we shall follow the proof of [9, Proposition 3]. Fix an $\varepsilon>0$ and choose an $r_{1} \in(0, r)$ such that

$$
\beta_{E_{r}}(z)<(1+\varepsilon) \beta_{D}(z), \quad z \in E_{r_{1}} .
$$

Combining the argument in the case $n \notin A$ from the previous proof and the estimates from Proposition 1, we may find an $r_{2} \in\left(0, r_{1}\right)$ such that if $z, w \in E_{r_{2}}$ and $\gamma:[0,1] \rightarrow D$ is a smooth curve for which $\gamma(0)=1$, $\gamma(1)=w$ and

$$
\int_{0}^{1} \beta_{D}\left(\gamma(t) ; \gamma^{\prime}(t)\right) d t \leq(1+\varepsilon) b_{D}(z, w),
$$

then $\gamma([0,1]) \subset E_{r_{1}}$. It follows that

$$
\begin{gathered}
b_{E_{r}}(z, w) \leq \int_{0}^{1} \beta_{E_{r}}\left(\gamma(t) ; \gamma^{\prime}(t)\right) d t \\
\leq(1+\varepsilon) \int_{0}^{1} \beta_{D}\left(\gamma(t) ; \gamma^{\prime}(t)\right) d t \leq(1+\varepsilon)^{2} b_{D}(z, w), \quad z, w \in E_{r_{2}} .
\end{gathered}
$$

To obtain (8), it remains to let $\varepsilon \rightarrow 0$.

So, $\lim _{\substack{z, w \rightarrow 1 \\ z \neq w}} \frac{b_{E_{r}}(z, w)}{b_{D}(z, w)}=1$. 
On the other hand, [6. Proposition 8] gives the estimates from Proposition 1 for $2 k_{D}$ instead of $\sqrt{2} b_{D}$. Then we obtain as above

$$
\lim _{\substack{z, w \rightarrow 1 \\ z \neq w}} \frac{\kappa_{E_{r}}(z, w)}{\kappa_{D}(z, w)}=1 .
$$

Now, the equality $b_{E_{r}}=\sqrt{2} k_{E_{r}}$ completes the proof.

\section{REFERENCES}

[1] Z. M. Balogh, M. Bonk, Gromov hyperbolicity and the Kobayashi metric on strictly pseudoconvex domains, Comment. Math. Helv. 75 (2000), 504-533.

[2] F. Forstneric, J.-P. Rosay Localization ot the Kobayashi metric and the boundary continuity of proper holomorphic mappings, Math. Ann. 279 (1987), 239-252.

[3] G. Herbort, Estimation of the Carathéodory distance on pseudoconvex domains of finite type, whose boundary has a Levi form of corank at most one, Ann. Polon. Math. 109 (2013), 209-260.

[4] M. Jarnicki, N. Nikolov, Behavior of the Carathéodory metric near strictly convex boundary points, Univ. Iag. Acta Math. XL (2002), 7-12.

[5] M. Jarnicki, P. Pflug, Invariant distances and metrics in complex analysis, de Gruyter Exp. Math. 9, de Gruyter, Berlin.

[6] N. Nikolov, Estimates of invariant distances on "convex" domains, Ann. Mat. Pura Appl. 193 (2014), 1595-1605.

[7] N. Nikolov, Comparison of invariant functions on strongly pseudoconvex domains, J. Math. Anal. Appl. 421 (2015), 180-185.

[8] N. Nikolov, P. Pflug, P. J. Thomas, Upper bound for the Lempert function of smooth domains, Math. Z. 266 (2010), 425-430.

[9] S. Venturini, Comparison between the Kobayashi and the Carathéodory distances on strongly pseudoconvex bounded domains in $\mathbb{C}^{n}$, Proc. Am. Math. Soc. 107 (1989), 725-730.

Institute of Mathematics and Informatics, Bulgarian Academy of Sciences, Acad. G. Bonchev 8, 1113 Sofia, Bulgaria

FaCUlty of Information Sciences, State University of Library Studies and Information Technologies, Shipchenski Prohod 69A, 1574 Sofia, BULGARIA

E-mail address: nik@math.bas.bg

Institute of Mathematics, Faculty of Mathematics and Computer Science, Jagiellonian University, Lojasiewicza 6, 30-348 Kraków, Poland

E-mail address: maria.trybula@im.uj.edu.pl 